\title{
Unequal Ageing: the Politics of Ageing As the Politics of Health Inequalities
}

\subsection{Introduction}

One major implication of the previous two chapters is that the politics of ageing is actually the politics of inequality - not a chimera of intergenerational inequality, but rather the inequalities that scholars of politics, social policy and health have long studied and understood (Lynch, 2020). As chapter two showed, one of the problematic assumptions of the 'ageing crisis' narrative is precisely the belief that older populations are homogeneous in their experience and outlook. This ignores the significant health inequalities which exist amongst older populations and overlooks the degree to which the costs of an ageing population are actually rooted in these inequalities. Without this framing, debates about intergenerational inequalities and the 'ageing crisis' are a distraction from both the deep social inequalities that exist in terms of gender, geography, race and ethnicity, socioeconomic status and the ways in which these social inequalities produce inequities in health. Intentional or not, to focus on intergenerational inequalities diverts attention from the real inequalities that shape people's lives and the politics of ageing and health.

These inequality-generating processes are not only located at one point in the life-course, of course. Health inequalities among older populations are the cumulative product of particular trajectories through the social spaces which create social and economic advantage and disadvantage. Indeed, one of the most profound implications of this emphasis on the 'ageing crisis' is that it has a tendency to obfuscate the continuing inequalities in who gets to be 'old' and who gets to enjoy good health in retirement. In this respect, the politics of ageing is more properly framed as the politics of inequality - and this cuts across generations.

In this chapter we draw out this argument by illuminating both the shape and scope of health inequalities across the life course and describing the causes of health inequalities. This is not an exhaustive 
overview of this large literature but instead we aim to provide a picture of how ignoring inequalities in health among children and the workingage population lays the foundation for the ageing crisis that concerns politicians and think tanks. We begin by examining inequalities in who gets to be old across a number of socio-demographic characteristics before offering a theory of the causes of health inequalities.

\subsection{Unequal Ageing: Who Gets to Be Old?}

Ageing and becoming 'old' are not universal experiences. There are significant social inequalities across the population in terms of life expectancy and disability-free life expectancy by gender, geography, race and ethnicity, socioeconomic status, etc. Health inequalities exist along many of the axes which divide and organize social life. There are geographical inequalities in health (e.g. by country, region or neighbourhood), and sex- or gender-based inequalities too. Inequalities in health cut across income, class, occupation and education, and they affect different racial groups. Health inequalities are systematic, avoidable and unfair differences in health (Whitehead, 1991).

\subsubsection{Gender Inequalities in Health}

In many countries women live longer than men - for example, life expectancy for women in Albania is over 4 years higher than for men (Albanian men 77.5 years, women 81.9 years); similarly, on average German women live $\sim 5$ years longer than American men $(83.2$ years compared to 78.4 years). Figure 1.2 in the introductory chapter shows how serious these inequalities can be, with the case of Lithuania representative of the many countries with dramatic gender inequalities in life expectancy.

Longer female life expectancies do not, however, mean better health. Women (particularly older women) also generally experience worse health: women get sick, men die (Doyal, 1995). For example, in France more men than women consider their health to be 'good' (70.2 per cent for men and 65.6 per cent for women). Limiting long-term conditions tend to be higher amongst older women than amongst older men. Women also have higher rates of mental ill-health, particularly in terms of depression and anxiety across all age groups. However, suicide rates are higher amongst men (Daykin \& Jones, 2008). Health differences 
between men and women are partly explained in biological terms (i.e. sex-based) and partly in social terms (i.e. gender-based). Sex refers to the biological differences between men and women, whilst gender is the social construction of sex-related roles and relationships - how men and women are expected to act within particular societies (Hill, 2016).

Research on differences in health between men and women focuses on the interaction of these two different elements, although the social tends to be privileged. For example, men's poorer longevity is ascribed by some to men's higher rates of risky health behaviours such as alcohol consumption and drug abuse, or to the fact that men are less likely than women to access health care services (Annandale \& Hunt, 2000). These are linked to gendered assumptions of what it means to be a 'man' within dominant forms of masculinity. Social factors are also important as women have less access to higher paid jobs and therefore less access to health-promoting resources. Employment is often gendered, with men and women therefore experiencing different working conditions. Women also continue to bear the brunt of the unpaid domestic labour and caring responsibilities. Institutional or structural sexism is also important (Homan, 2019). For example, studies have shown that in countries where men and women are more equal, the health of both men and women tends to be better (Reeves et al., 2016; Van de Velde et al., 2013).

\subsubsection{Ethnic Inequalities in Health}

Health also varies by race and ethnicity (Nazroo \& Williams, 2006). For example, in the Netherlands all-cause mortality rates are more than 1.2 times higher for Turkish, Surinamese and Antillean/Aruban males even after adjusting for other factors (Bos et al., 2004). In the USA African-Americans have a life expectancy four years lower than that of White Americans (Arias, 2011), whilst indigenous populations in the USA, Canada, New Zealand and Australia experience life expectancies of seven to twelve years less than their non-indigenous counterparts (Hill, 2016).

Non-white populations tend to suffer a greater burden of morbidity as well. American Whites spend a greater share of their years free of activity limitations (Molla, 2013). Importantly, morbidity and mortality disparities between Whites and African-Americans shrink and gradually disappear over the life-course. Among the very old, African-Americans 
appear to enjoy a health advantage over Whites. This phenomenon, referred to as the racial morbidity and mortality crossover, is the subject of an ongoing scientific debate. While some scholars have hypothesized that the crossover may be a measurement artefact, others have argued that adverse life-course selection translates to better overall health of the oldest African-Americans.

Sociologists and social epidemiologists have persuasively demonstrated that a large share of today's observed racial and ethnic differences in health are due not to innate biological differences between members of different racial and ethnic groups but to social and environmental factors. For example, Cooper and colleagues (2005) showed that while US Whites have lower prevalence of hypertension than African-Americans, they have greater prevalence of hypertension than Blacks living in Africa. In contrast, African-Americans have higher hypertension rates than populations of predominantly White European countries, such as Sweden and Italy, but lower than others, including Germany and Finland. Because wealth and socioeconomic resources tend to be unevenly distributed in racially and ethnically stratified societies socioeconomic status interacts with racial and ethnic disparities. In the US context, the median net worth of a White household is nearly nine times greater than that of a Black household and five times greater than that of a Hispanic household. Asian-American households' median net worth exceeds that of Whites by 1.4 times (Eggleston \& Munk, 2019). The large disparities between people of different races, partly reflect economies and social policies in which socioeconomic resources are distributed by race.

Prior research has shown that even after adjusting for socioeconomic status, morbidity and mortality disparities by race/ethnicity remain. Perhaps surprisingly, racial health disparities are larger among the more privileged than the less privileged, whose outcomes are more uniform (Braveman et al., 2010). Racism and racial discrimination likely account for a share of the health outcome differences between similarly advantaged White and non-White populations (D. R. Williams et al., 2019). As an upstream cause of disadvantage, racism and racial discrimination may influence health through multiple pathways. For example, racial segregation can limit access to healthful neighbourhoods and desirable housing options, and has usually been linked to worse health in Blacks, including worse pregnancy outcomes and increased mortality (Kramer \& Hogue, 2009). Discrimination has been shown to reduce access to goods, services and health care in multiple national contexts 
(Ben et al., 2017). In addition, experiences of discrimination may also have a less immediately apparent effect - they are likely to increase individual stress and translate to higher allostatic load (Allen et al., 2019), which has been connected to the emergence of multiple chronic conditions (McEwen \& Stellar, 1993).

\subsubsection{Socioeconomic Inequalities in Health}

Socioeconomic status is a term that refers to occupational class, income or educational level (Bambra, 2011). Across Europe, people with higher occupational status (e.g. professionals such as teachers or lawyers) have better health outcomes than those with lower occupational status (e.g. manual workers). Similarly, people with a higher income or tertiary-level education have better health outcomes than those with a low income or no educational qualifications (Bambra, 2016). Current levels of health inequalities in Europe are depicted in Figures 5.1, 5.2 and 5.3.

Figure 5.1 shows inequalities in general health by income group. Good health increases with income in all countries (Forster et al., 2018). At the EU level, 60.0 per cent of people with a low income report to be in good health compared to 78.3 per cent of people with a high income: a difference of over 18 percentage points. Figure 5.1 also shows how inequalities in general health vary across Europe. For example, the health gap is 20 percentage points in Ireland and Portugal but almost 40 in Lithuania.

Figure 5.2 shows educational inequalities in long-term chronic diseases (non-communicable diseases) (McNamara et al., 2017). Again, there are clear inequalities across Europe. For instance, someone with a low education is over three times more likely than someone with a tertiary education to report depression. As Figure 5.2 also indicates, educational inequalities in Europe are highest for depression, diabetes and obesity.

There are also socioeconomic inequalities in health-related practices: rates of smoking and alcohol consumption are higher in lower socioeconomic groups, whilst rates of physical activity and consumption of fruit and vegetables are lower (Huijts et al., 2017). For example, as Figure 5.3 shows, smoking follows a clear social gradient: in all European countries except Portugal the likelihood of smoking daily is higher for individuals with a primary or secondary education than for their more highly educated peers (Huijts et al., 2017). 


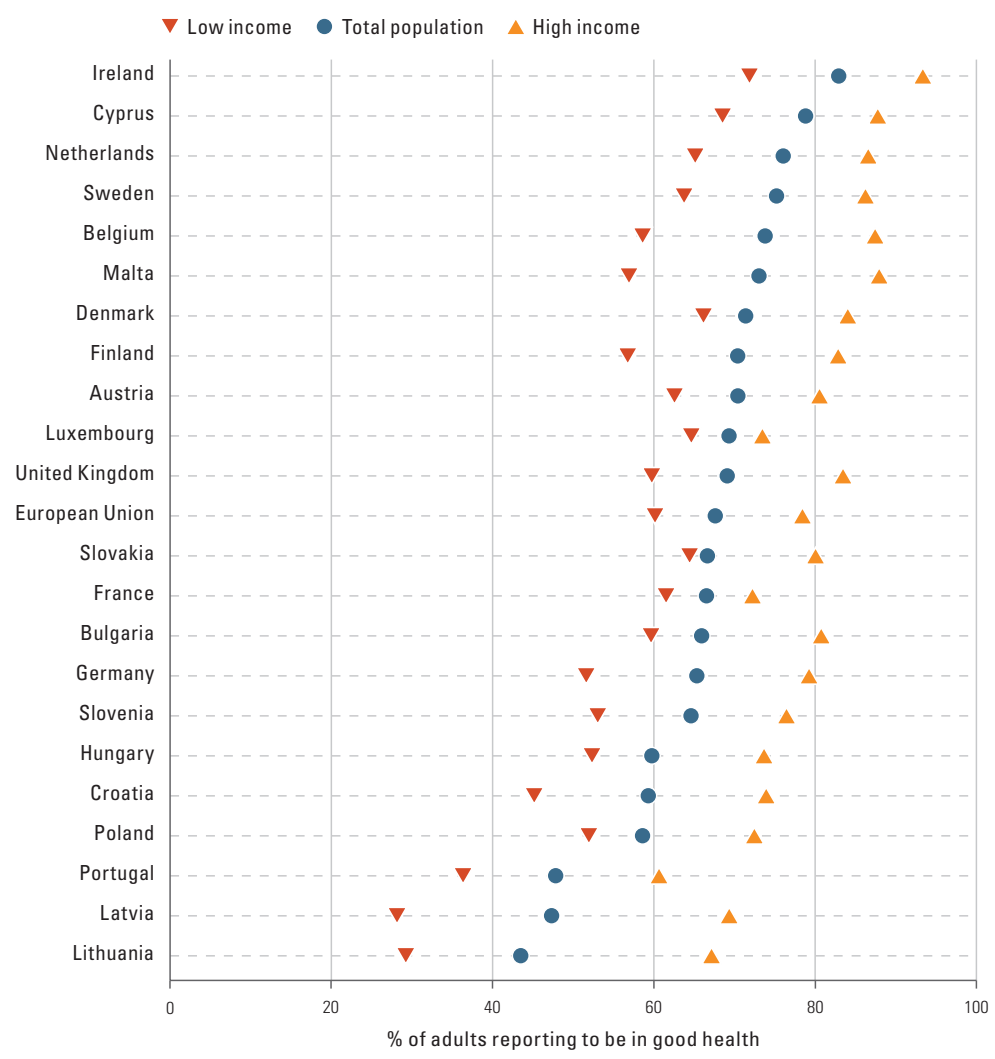

Figure 5.1 Income inequalities in self-reported health across Europe, 2016.

Notes: Data refer to 2016.

Source: Forster et al., 2018, reproduced with permission of authors

\subsubsection{Geographical Inequalities in Health}

It is well known that health varies between countries. Most notably, there are considerable differences between the richest and the poorest European countries. For example, average life expectancy for men and women in countries like France is high (over 83 years), while in Latvia or Lithuania it is significantly lower ( 75 years in both). The USA, the richest country in the world, has more than four years less life expectancy than Japan and an infant mortality rate that is three times greater than that of Iceland (Schrecker \& Bambra, 2015). This has led to discussions of a 'US health disadvantage' whereby the USA has worse 


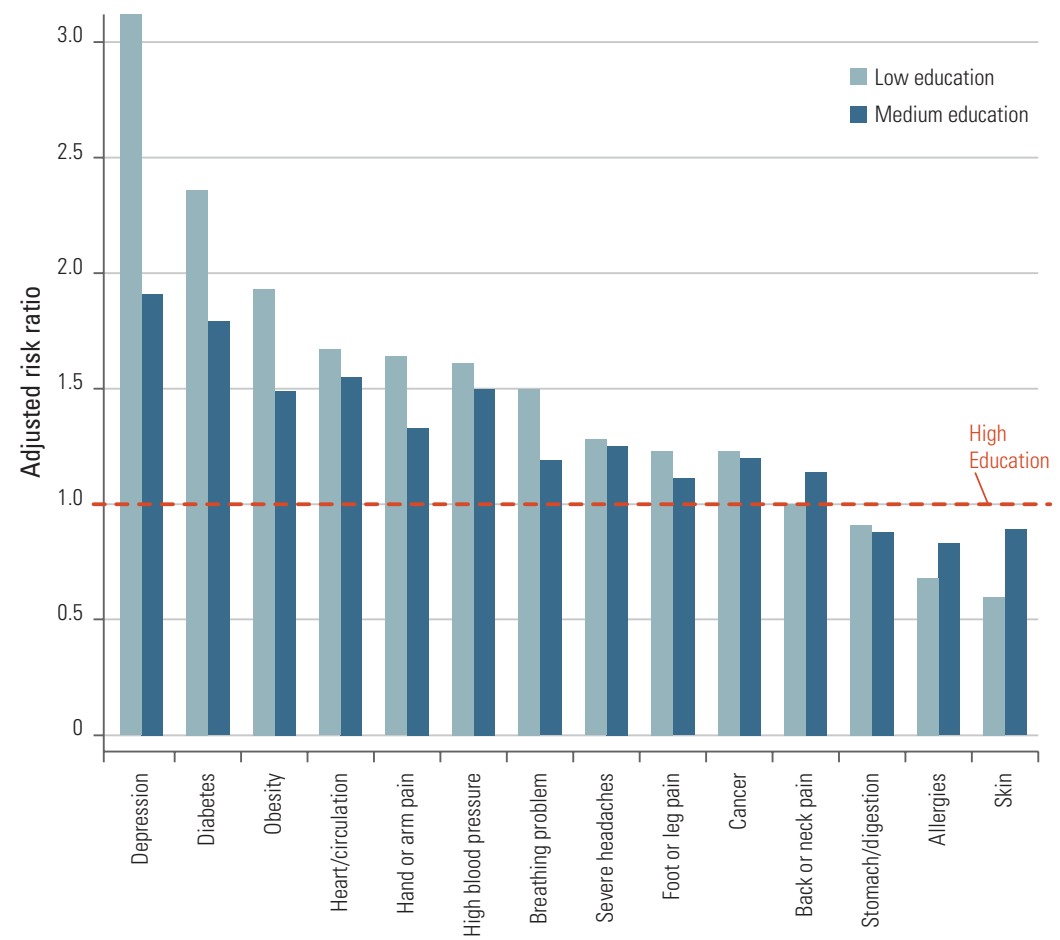

Figure 5.2 Educational inequalities in non-communicable diseases in Europe.

Notes: Age-adjusted risk ratios estimate the probability of self-reporting a particular NCD for individuals with low and medium education vis-à-vis highly educated people.

Source: Forster et al., 2018, reproduced with permission of authors

outcomes across a number of key health outcomes (such as obesity or heart disease) than other comparable wealthy countries (US National Research Council \& US Institute of Medicine, 2013).

Within countries, there are also regional inequalities in health. For example, in Belgium in 2016 the standardized death rate per 100,000 was 886 deaths in the province of West Vlaanderen but in Hainaut it was 1,161 deaths (Forster et al., 2018). These regional inequalities in health are evident in all other countries. By way of example, Figure 5.4 shows regional inequalities in life expectancy across Europe for men and women combined. It shows that across Europe, all countries experience 


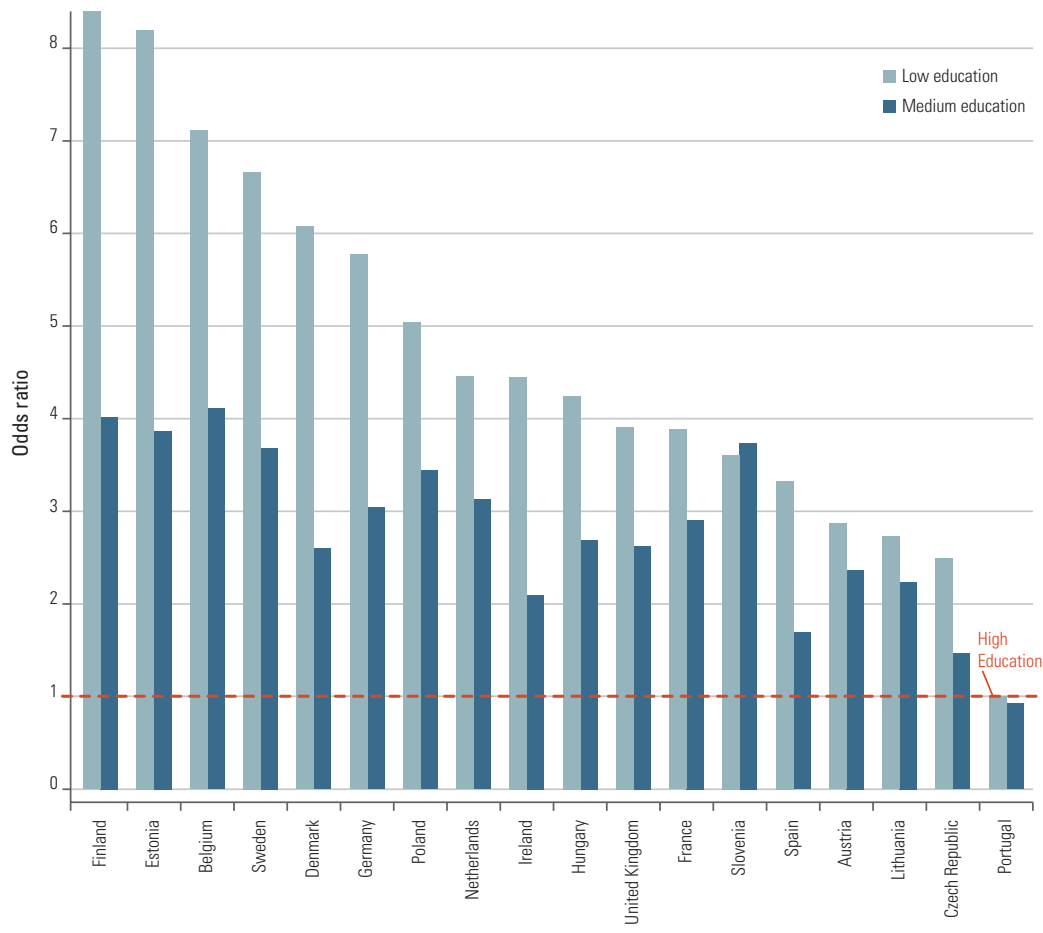

Figure 5.3 Probability of smoking by educational status.

Notes: Age- and sex-adjusted odds ratios represent the probability of being a daily smoker given low or medium education, respectively, relative to individuals with high education.

Source: Forster et al., 2018, reproduced with permission of authors

unequal health across their constituent regions. These patterns also exist outside Europe as, for example, in China, where average life expectancy is over five years less in the northern provinces than in the southern ones.

The most striking geographical inequalities in health, though, are those that exist between neighbourhoods, with neighbourhoods that are the most deprived (measured in terms of income, employment, health, education, crime, access to services and living environment) having worse health than those that are less deprived - and this follows a spatial gradient with each increase in deprivation resulting in a decrease in average health. In England the gap between the most and least deprived areas is around nine years average life expectancy and nineteen years average 


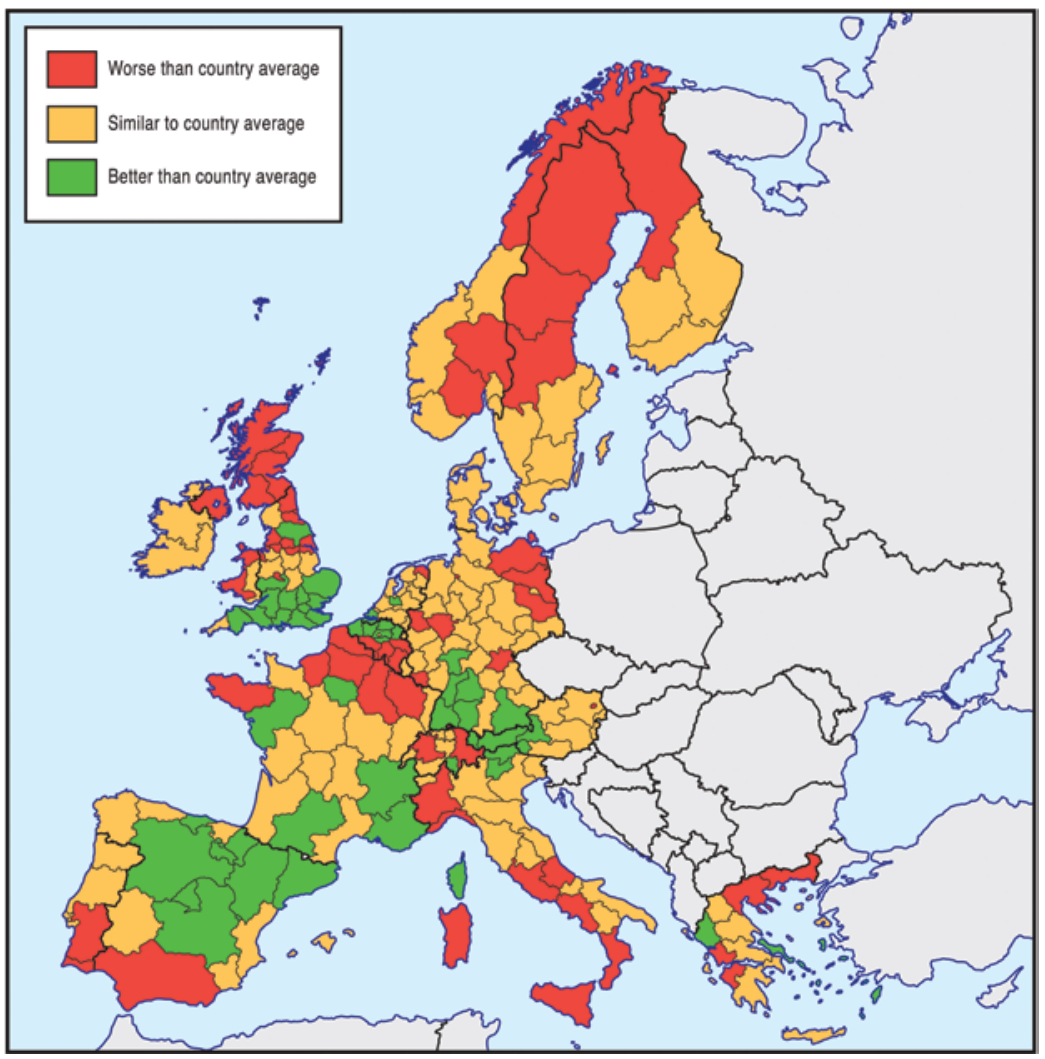

Figure 5.4 Average life expectancy by European region for men and women. Source: reproduced from Bambra, 2016, with permission of Policy Press

healthy life expectancy for men and around seven and nineteen years respectively for women (ONS, 2018). In some cases these deprived and affluent areas with such differences in health outcomes can be located very closely together - indeed just a few miles apart, as with London, where life expectancies along a single Underground line, the Bakerloo Line, vary dramatically (Bambra, 2016).

\subsubsection{Intersectional Inequalities}

Health inequalities are also intersectional. Intersectionality is a way of looking at multiple influences on health (Gkiouleka et al., 2018). It focuses on how socioeconomic status, ethnicity and gender are 
experienced not separately but in combination and that we all have different aspects of social identity that coexist with one another. Focusing on any one element of inequality, such as patriarchy or White supremacy, risks obscuring the ways in which different inequalities interact.

Intersectionality therefore looks at the 'axes of inequality', particularly socioeconomic status, gender and ethnicity together. It also considers gender and ethnicity as social factors rather than simply demographic ones, viewing them as socially structured, constructed and experienced. So, for example, health differences between men and women arise not just because of biological differences but as a result of the social construction of sex-related roles and relationships (gender). Likewise, ethnic inequalities in health can arise through racism, with ethnic minority groups more likely to experience discrimination personally, institutionally (systematic exclusion and disadvantage) and economically. Health is therefore affected by each of these axes of inequality (Hill, 2016). Intersectionality therefore draws recognition to the fact that any individual has 'such multiple aspects of identity with relevance for their relationships with others and with the structures and systems of power - and, therefore, for their health'. Intersectionality looks at all aspects of the individual and how they experience the various aspects of their social identity within particular contexts, and with specific health effects. Black men, for example, have far poorer health than White women. These inequalities also intersect with age. While older people have poorer health than younger people, not all older people experience poor health in the same way. For example, ethnic minorities in France aged 65 and over were almost twice as likely $(\sim 40 \%)$ to report a heart or circulation problem than those who were members of the ethnic majority $(\sim 21 \%)$.

\subsubsection{Trends in Health Inequalities}

These intersecting inequalities have become crucial to political debates across high-income countries because they are behind the stagnation of life expectancy in some contexts. For example, stagnating life expectancy in the UK has been driven by a mix of rising mortality among both the elderly and, more surprisingly, those of working age. Perhaps more striking, inequalities in life expectancy have tragically risen in the last few years, according to recently released figures from the Office for National Statistics (ONS, 2019). There are also signs that inequalities in 
infant mortality rates may also be increasing. This is not only because of greater improvements among the better-off but because the poorest have seen real declines. In the most deprived parts of England, female life expectancy at birth fell by almost 100 days between 2012-2014 and 2015-2017. Men in the poorest areas saw no improvements, while those in the richest parts of the UK continued to see their life expectancies improve. This is true among the elderly too.

In the USA all-cause mortality rates stopped falling around the turn of the twenty-first century (Case \& Deaton, 2015). The halt of mortality improvements has been traced to increased mortality in middle-aged non-Hispanic Whites. While mortality of Whites in the youngest and oldest ages has continued to decline, as have mortality rates for Blacks and Hispanics, their gains were overshadowed by rapid growth in what Case and Deaton refer to as deaths of despair in middle-aged Whites: drug overdoses, suicides and alcohol-related liver mortality. The mortality rate increase was accompanied by worsened self-rated health and greater reports of chronic pain (Case \& Deaton, 2015). The social causes underlying the mortality rate increase in middle-aged Whites are the subject of ongoing discussion. Case and Deaton argue that they are symptoms of a cumulative disadvantage of working class Whites, including weakening labour market and social structures, as well as falling marriage rates and lower participation in organized religion (Case \& Deaton, 2017). Others have leaned towards more macroeconomically oriented explanations (Hollingsworth et al., 2017) or argued that the main culprit behind increased drug-related mortality is increased availability of drugs (Ruhm, 2018). We explore this further in Chapter 6.

These trends in terms of stalling and indeed falling life expectancy in the UK and Europe are also reflected in terms of trends in health inequalities. Since the global financial crisis of 2007-8, Europe has experienced considerable economic, social and political upheaval. Across Europe the economic recovery has been weak and inequitable: unemployment has remained high, particularly amongst young adults and those with low skills, wages have stagnated and living standards have declined. Relatedly, health inequalities have also increased. Whilst average life expectancy at birth in the European Union increased from 79.4 in 2008 to 81.0 in 2016, these increases were smaller amongst men and women with a lower level of education (Forster et al., 2018). For example, in Denmark the difference in life expectancy at age 30 between men with a low education and men with a tertiary education rose from 4.8 years 
to 6.4 years. The respective gap for women increased from 3.7 years to 4.7 years. So, whilst the health of everyone in Europe has improved over the last decade, more privileged groups have benefited the most, leading to increased health inequalities in some countries (Mackenbach et al., 2016).

\subsubsection{COVID-19 Pandemic and Health Inequalities}

These social inequalities in health by gender, geography, race and ethnicity, and socioeconomic status are evident across all health conditions, from mental health through to chronic diseases such as diabetes and heart disease, and even in infectious diseases including Covid-19 (Bambra et al., 2020; Bambra et al., 2021). There is clear evidence of social inequalities in COVID-19 infection and mortality rates from Spain, the USA and the UK. For example, intermediate data published by the Catalonian government in Spain in April 2020 suggests that the rate of COVID-19 infection is six or seven times higher in the most deprived areas of the region compared to the least deprived (Catalan Agency for Health Quality and Assessment, 2020). Similarly, in preliminary USA analysis, Chen and Krieger (2020) found area-level socio-spatial gradients in confirmed cases in Illinois and New York City, with dramatically increased mortality risk observed among residents of the most disadvantaged counties. Official national data in England and Wales found that coronavirus (COVID-19)-related deaths were twice as high in the most deprived neighbourhoods (55 per 100,000 population) than in the most affluent neighbourhoods (25 per 100,000 population) (ONS, 2020).

In regards to ethnic inequalities in COVID-19, data from England and Wales found that people who are Black, Asian and minority ethnic (BAME) accounted for around 35 per cent of 5,000 critically ill COVID19 patients (in the period ending 16/4/2020) - although they only make up 14 per cent of the UK population (ICNARC, 2020). Similarly, there are large racial inequalities in COVID-19 infections and deaths in various US states and municipalities. For example, in Chicago (in the period ending 17/04/20), 60 per cent of COVID-19 deaths were amongst Black residents and the COVID-19 mortality rate for Black Chicagoans was 35 per 100,000 population compared to 8 per 100,000 population amongst White residents (City of Chicago, 2020). Gender also appears to be a risk factor for COVID-19 symptom severity and mortality, with data from many countries showing that mortality rates are much higher 
amongst men. For example, in March and April 2020 in England and Wales men made up 70 per cent of hospitalized cases and around 60 per cent of confirmed COVID-19-related deaths (ICNARC, 2020).

As the pandemic progresses, there will likely be an interaction of race, gender and socioeconomic inequalities, given the intersectionality of multiple aspects of social disadvantage (Gkiouleka et al., 2018).

\subsection{What Causes Health Inequalities?}

The explanations for different forms of health inequalities are rooted in the connection between health-related practices, material resources and institutions. These intersect with other features of the social world, such as socioeconomic status, place, gender and ethnicity. In the sections below, we focus on social position and place rather than gender and ethnicity. This is not because gender inequalities and ethnic inequalities are unimportant, but we focus on those which are most deeply connected to the ageing process generally and to our policy analysis in Chapter 6.

\subsubsection{Material Resources: the Social Determinants of Health}

'Materialist' explanations focus on the conditions which influence our access to health-enhancing goods, which limit our exposure to risk factors. Economic resources, for example, are one of these conditions because they can determine our ability to afford good quality services (e.g. hospitals, schools, transport infrastructure and social care) but also allow people to avoid materially harmful circumstances (e.g. poor housing, inadequate diet, physical hazards at work, environmental exposures). Fundamental cause theory (Phelan et al., 2010) is premised on the idea that money is fungible and therefore can be used to preserve inequalities, so if we address one specific inequality (e.g. lack of access to green spaces) overall health might improve as a result but income inequalities will lead to some other measurable health inequality increasing. Furthermore, runs the theory, new technology will often increase inequality: the benefits of wearable devices, for example, will accrue to better-off people first, which means that regardless of the intentions of the creators or the effect of the technology among users, most new health technologies will increase health inequalities.

The conditions which structure the social worlds in which we live, work, mature and die are commonly termed the 'social determinants 
of health'. Beside income, they include working conditions, housing and neighbourhood, labour market activity and access to certain goods and services, such as health care. Our social position in the world is a complex agglomeration of all of these aspects of our lives and so in a very meaningful sense people in different social positions inhabit quite distinct social and epidemiological life worlds.

One way in which our social position in the world influences health is through shaping our practices - how we live our lives. Health inequalities are grounded in everyday practices, both chosen and habitual. These practices are often called health behaviours (a term which can imbue social action with a degree of intentionality). However, social action is not always intentional and so we call them practices because these 'behaviours' often bear the hallmarks of a particular mode of engaging with the world which relies on pre-verbal understanding of what is appropriate in a given setting. Health-related practices, then, are ways people spend their time and also forms of consumption that affect health, including diet, exercise and other forms of consumption such as tobacco use. Practices are, of course, not solely determined by individual preferences. In fact, when we articulate our preferences we are usually expressing post-hoc justifications for habits that have not necessarily been subject to rational evaluation (Jerolmack \& Khan, 2014). What we consume and how we spend our time is shaped by our social position because the 'worlds' we inhabit - whether we have a high income or not - make possible some forms of social action whilst making other practices more difficult. By cultivating distinct sets of practices, these 'worlds' can, as described above, have implications for health and health inequalities. When Mexicans leave cans of CocaCola on the graves of their ancestors on 'The Day of the Dead', they are revealing the value their community (or their social position in the world) places on specific forms of consumption.

The social nature of practices influences the size and shape of health inequalities and partially explains why the degree to which practices (or behaviours) influence health inequalities varies within and between countries over time. This is because practices are not inherently linked with inequalities per se. That is, specific practices only generate health inequalities if there are inequalities in who performs those practices. To come back to tobacco, smoking is a social practice which reflects gender roles, social class structures and income inequalities (Marron, 2017). So, as inequalities in smoking have decreased in Europe in 
recent decades, smoking has become a less important determinant of health inequalities. Crucially, there are important gender differences here because this decline has primarily been observed in men. This is not true of women, however, because inequalities in smoking among women have in fact increased (Gregoraci et al., 2017).

Practices differ between and within age groups. In 2014 the prevalence of 'current smokers' across the twenty-eight European member states was 32.6 per cent among 25-34 year olds and 26.7 per cent of $45-64$ year olds. This $\sim 6$ percentage point gap is actually smaller than the 7 percentage point difference between the poorest income quintile and the richest quintile. In fact, the really striking difference is between income quintiles within specific age groups. There is a 13 percentage point gap in the prevalence of 'current smokers' between the poorest and the richest income quintiles among 25-34 year olds, and an 11.1 percentage point gap between the poorest and the richest income quintiles among those aged 55-64. These are differences that are going to have a profound influence on the costs of ageing in the future, and they are differences driven by the social determinants of health.

Finally, the social determinants of health may influence health outcomes through psychosocial pathways (Marmot, 2004). Beyond the materialist and practice-based explanations, the psychosocial is concerned with the psychic experience of social inequalities or other forms of social exclusion (Greer, 2018; Pickett \& Wilkinson, 2010, 2015). The experience of discrimination or racism, for example, has profound negative effects on the health of the dominated racial group (D. R. Williams et al., 2019). Discrimination in the labour market leaves dominated racial groups in economically precarious positions, locked out of the mechanisms that ensure wealth accumulation (Shapiro, 2017). Self-reported discrimination is also associated with poorer mental health and (albeit less clearly) physical health as well (D. R. Williams et al., 2019).

A study using data from Norway compared the relative contribution of practices, material resources and psychosocial explanations (alongside biomedical factors) of socioeconomic (educational and income) inequalities in mortality. It concluded that material factors were the most important in explaining income inequalities in mortality whilst psychosocial and behavioural factors were the most important in explaining educational inequalities (Skalická et al., 2009). People exposed to one sort of factor (e.g. material) may also be exposed to 
another (e.g. psychosocial) and have interacting influences on their health. So, these explanations of health inequalities are intersecting rather than competing. Indeed, people of higher socioeconomic status (be it higher education levels, higher income or higher occupational class) have access to better resources, money, knowledge, prestige, power and beneficial social networks, whilst those of lower socioeconomic status (lower income, education or occupational class) have less access to such things. People of higher socioeconomic status also have access to better contexts (as shall be described in the next section) in terms of workplaces and neighbourhoods (Phelan et al., 2004). The health advantage of having a higher socioeconomic status is robust to varying degrees over time and place such that, whilst the clinical and proximal causes of socioeconomic inequalities in health have changed, the gradient still exists. For example, in the nineteenth century the leading causes of death were fevers and consumption, whilst in the twentieth and early twenty-first centuries the main causes of death are cancers and cardiovascular disease. Socioeconomic inequalities existed in the nineteenth century as they do today - despite the diversity of the actual causes of death. This is because high socioeconomic status in the nineteenth century enabled people to have better sanitation or to live away from polluting factories, for example, whilst in the twentieth and twenty-first centuries it enables better health-related practices or access to healthier work environments. People of high socioeconomic status use their resources to adapt to the disease threat of the day and gain a health advantage over those of lower socioeconomic status who do not have the resources to adapt. This has led to socioeconomic status being called a 'fundamental cause' of ill health - and ageing - which persists despite competing causes of death (Link \& Phelan, 1995).

\subsubsection{Explaining Geographic Inequalities in Health}

Building on explanations of socioeconomic inequalities in health, researchers have traditionally explained geographical inequalities in health in terms of the effects of compositional (who lives here?) and contextual (what is this place like?) factors (Bambra, 2016). The compositional explanation asserts that the health of a given area, such as a town, region or country, is a result of the characteristics of the people who live there (individual-level demographic, health-related practices 
and socioeconomic factors). If more people smoke in a given area, then we would expect that area to have poorer health.

The contextual explanation, by contrast, argues that area-level health is also in part determined by the nature of the place itself in terms of its economic, social and physical environment. Health differs by place because it is also determined by the economic, social and physical environment of a place: poor places lead to poor health. Place mediates the way in which individuals experience social, economic and physical processes on their health: places can be salutogenic (health promoting) or pathogenic (health damaging) environments - place acts as a health ecosystem. These place-based effects can also be seen as the collective effects of the social determinants of health. That is, the socioeconomic position of the people who live in your community will impact your health irrespective of your own socioeconomic position. To capture these contextual effects, researchers commonly look at area poverty rates, unemployment rates, wages and types of work and employment in the area. The mechanisms whereby the economic profile of a local area impacts on health are multiple. For example, it affects the nature of work that an individual can access in that place (regardless of their own socioeconomic position). It also impacts on the services available in a local area, as more affluent areas will attract different services (such as food available locally or physical activity opportunities) than more deprived areas as businesses adapt to the different consumer demands in each area (see access to services in the opportunity structures section below). Area-level economic factors such as poverty are a key predictor of health, including cardiovascular disease, all-cause mortality, limiting long-term illness and health-related behaviours (Macintyre, 2007).

Places also have social aspects which impact on health. For example, local environments can shape our access to healthy - and unhealthy goods and services, thus enhancing or reducing our opportunities to engage in healthy or unhealthy behaviours such as smoking, alcohol consumption, fruit and vegetable consumption or physical activity. Alongside access to services, another social aspect of place is collective social functioning. Collective social practices that are beneficial to health include high levels of social cohesion and social capital within the community. Social capital - 'the features of social organisation such as trust, norms, and networks that can improve the efficiency of society by facilitating coordinated actions' (Putnam, 1993, 167) - has been put forward as a social mechanism through which place mediates 
the relationship between individual socioeconomic status and health outcomes (Hawe \& Shiell, 2000). Some studies have found that areas with higher levels of social capital have better health, such as lower mortality rates, self-rated health, mental health and health behaviours. More negative collective effects can also come from the reputation of an area (e.g., stigmatized places can result in feelings of alienation and worthlessness) or the history of an area (e.g., if there has been a history of racial oppression). Residents of stigmatized places can also be discredited by association with these place characteristics. A notable and documented case of such place-based stigma is Love Canal, New York - the location of a toxic waste dump. Research has shown that such place-based stigma can result in psychosocial stress and associated ill health, alongside feelings of shame, on top of the physical health effects of air pollution such as respiratory disease (Airey, 2003).

Finally, place can also affect health through the nature of the physical environment (Marmot et al., 2008). There is a sizeable literature on the positive health effects of access to green space, as well as the negative health effects of waste facilities, brownfield or contaminated land, as well as air pollution (Bambra, 2016). One (in)famous example of the latter is the so-called Cancer Alley - the 87-mile stretch in the American state of Mississippi between Baton Rouge and New Orleans, the home of the largest petrochemicals site in the country (Markowitz \& Rosner, 2003). In 2016 it was estimated that air pollution levels in London accounted for up to 10,000 unnecessary deaths per year (Walton et al., 2015). Another example of how the physical environment of areas varies is in respect to land pollution. A study found that in the American city of Baltimore mortality rates from cancer, lung cancer and respiratory diseases were significantly higher in neighbourhoods with larger amounts of brownfield land (Litt et al., 2002). The literature has also established the role of natural or green spaces as therapeutic or health-promoting landscapes. So, for example, studies have found that walking in natural, rather than urban, settings reduces stress levels and people residing in green areas report less poor health than those with less green surroundings (Maas et al., 2006). The unequal socio-spatial distribution of the environmental deprivation has also led to commentators developing the concept of environmental justice (Pearce et al., 2010). The fact that more deprived neighbourhoods are more likely to have air and land pollution and less likely to have green space can be seen as an aspect of social injustice (Pearce et al., 2010). 
More recently, it has been acknowledged that the compositional and the contextual approaches are not mutually exclusive and that the health of places results from the interaction of people with the wider environment (relational approach) (Cummins et al., 2007). Indeed, separating them is an oversimplification and ignores the interactions between these two levels (Macintyre et al., 2002). The characteristics of individuals are influenced by the characteristics of the area. For example, occupational class can be determined by local school quality and the availability of jobs in the local labour market, or children might not play outside due to not having a private garden (a compositional resource), because there are no public parks or transport to get to them (a contextual resource) or because it might not be seen as appropriate for them to do so (contextual social functioning) (Macintyre et al., 2002). Similarly, areas with more successful economies (e.g. more high-paid jobs) will have lower proportions of lower socioeconomic status residents.

Further, the collective resources model suggests that all residents, and particularly those on a low income, enjoy better health when they live in areas characterized by more/better social and economic collective resources. This may be especially important for those on a low income as they are usually more reliant on local services. Conversely, the health of poorer people may suffer more in deprived areas where collective resources and social structures are limited, a concept known as deprivation amplification: the health effects of individual deprivation, such as lower socioeconomic status, can therefore be amplified by area deprivation (Macintyre, 2007).

These insights have profound implications for thinking about healthy ageing because the scarring effect of being born into a deprived community can linger long after people have moved away. Indeed, for the many people who stay in those communities, they are exposed to a series of disadvantages that impinge on their ability to live healthy lives. As Cummins and colleagues argue, 'there is a mutually reinforcing and reciprocal relationship between people and place' which will structure how the costs associated with ageing are distributed geographically (Cummins et al., 2007, 1826). As we have already argued, ageing is not costly for everyone, but it is particularly costly for those communities where people spend more years in poor health. These negative health effects are experienced by everyone in those communities, but they are most keenly felt by those with the greatest individual disadvantage. It is already becoming clear that the costs of social care are not evenly 
distributed geographically, and such inequalities may widen if the upwardly mobile continue to move to major urban centres to access well-paid, more prestigious jobs.

\subsection{Beyond the Social Position and Place: the Political Economy Approach}

Socioeconomic and spatial inequalities are not random chance, however, because they are rooted in political and economic institutions (Besley \& Kudamatsu, 2006; Greer et al., 2018; Lundberg, 2008). In 2014 the Lancet-University of Oslo Commission on Global Governance for Health put forward the concept of the 'political determinants of health', insisting that 'construing socially and politically created health inequities as problems of technocratic or medical management depoliticises social and political ills' (Ottersen et al., 2014, 636). The political economy approach argues that the social determinants of health (e.g., socioeconomic position and place) are themselves shaped by macro level structural determinants: politics, the economy, the (welfare) state, the organisation of work and the structure of the labour market (Bambra, 2011; Barnish et al., 2018) and that population health is shaped by the 'social, political and economic structures and relations' that may be, and often are, outside the control of the individuals they affect (Bambra et al., 2005; Krieger, 2001). Politics is understood here in its broadest terms as 'the process through which the production, distribution and use of scarce resources is determined in all areas of social existence' (Bambra et al., 2005), not simply as the actions of governments or political parties. Public health and health inequalities are thus considered to be politically determined with patterns of disease 'produced, literally and metaphorically, by the structures, values and priorities of political and economic systems ... Health inequities are thus posited to arise from whatever is each society's form of social inequality, defined in relation to power, property and privilege' (Krieger et al., 2013). In other words, why some places and people are consistently privileged whilst others are consistently marginalized is a political choice - it is about where the power lies and in whose interests that power is exercised. Political choices can thereby be seen as the causes of the causes of the causes of inequalities in health, both socioeconomic and spatial (Bambra, 2016).

To be more concrete, we can think of economic institutions as being concerned with the rules that govern who has access to the wealth 
produced by society, how the means of producing that wealth are organized, and the rules that govern competition in local and global markets. Taxes and transfers that pay for pensions, for example, can affect access to health care, and recent reductions in pension generosity in Europe are associated with rising inequalities in unmet medical need because the poorest experience reduced ability to afford health care whilst the richest experience no change (Reeves et al., 2017b). Minimum wage regulations constrain wage negotiations at the bottom of the income distribution and a number of papers have recently observed that the introduction of minimum wages improves mental health (Horn et al., 2017; Lenhart, 2017; Reeves, 2017b). Employment regulations that decommodify the labour market relations are associated with better health outcomes and lower absolute inequalities in health than countries which rely on more commodified market relationships (Beckfield \& Krieger, 2009). Trade policy (Jarman, 2019; Jarman \& Greer, 2010), which structures the rules that govern competition, may also influence health inequalities because they affect the social determinants of health (Barlow et al., 2017a; Blouin et al., 2009; Madureira Lima \& Galea, 2018). For example, trade agreements alter food environments (Barlow et al., 2017b; Owen \& Wu, 2007; Schram et al., 2015), expose some groups to long-term economic shocks (Rodrik, 2012) and may even harm infant mortality (Barlow, 2018).

Political institutions are also commonly 'listed alongside ... other fundamental causes' of health, albeit often without elaborating on 'what these political forces might be [or] how they operate' (Torres \& Waldinger, 2015). The theoretical reasoning connecting political institutions and health is relatively 'straightforward' (Bollyky et al., 2019). Political institutions influence population health because countries with free and fair elections, for example, make politicians more responsive to the preferences of citizens and this could influence policy decisions and the structure of economies, both of which may impact health and wellbeing (Kudamatsu, 2012; Wang et al., 2018; Wigley \& AkkoyunluWigley, 2011). Political institutions affect decision-making (Hill \& Leighley, 1992; Lijphart, 1997) because 'politicians and officials are under no compulsion to pay much heed to classes and groups of citizens that do not' get to participate in decision-making processes (Key \& Heard, 1949). When political institutions systematically exclude some groups from decision-making, their health is likely to suffer because the concerns of excluded groups may be overlooked (Fowler, 2015; Geys, 
2006; Krieger et al., 2014; Lijphart, 1997), even when those in power might be sympathetic to the policy preferences of the under-represented (Pontusson \& Rueda, 2010). Put simply, political institutions govern who has voice and this may affect health. While a number of studies are beginning to document the health effects of political institutions (Bollyky et al., 2019; Wigley \& Akkoyunlu-Wigley, 2011), this work is still in its infancy (Beckfield, 2018).

In this sense, patterns of health and disease are produced by the structures, values and priorities of political and economic systems (Krieger, 2001). Geographical patterns of health inequalities, at least in part, are shaped by the wider political, social and economic system and the actions of the state (government) and international level actors (Bambra, 2019). Politics and the balance of power between key political groups - notably labour and capital - determine the role of the state and other agencies in relation to health and whether there are collective interventions to improve health and reduce health inequalities, and also whether these interventions are individually, environmentally or structurally focused. In this way, politics (broadly understood) is the fundamental determinant of our health divides because it shapes the wider social, economic and physical environment and the social and spatial distribution of salutogenic and pathogenic factors both collectively and individually (Bambra, 2016).

\subsection{Conclusion}

This chapter has provided a brief overview of the different types of health inequalities that exist in all high-income countries and we have described some of the drivers of these inequalities in mortality and morbidity. The message of this chapter is straightforward: the politics of ageing is actually the politics of inequality. In other words, debates about the 'ageing crisis' could, in our estimation, be reframed as debates about health inequality. There are two reasons we hold this view.

Ageing is not a uniform process. Some adults live long and healthy lives and others do not. Many sadly never even get to reach 'old age', as defined by traditional metrics, while others suffer many years of pain and limited capacity as they live with chronic illnesses which constrain their ability to enjoy the kinds of lives they want to live. Unfortunately, such differences are not explained by luck alone. The substantial health inequalities that exist among the elderly are structured according to other 
inequalities in the social determinants of health. Differences in income, education and social background shape our everyday practices, such as whether we smoke or what we eat, and these affect our life chances. Beyond these individual characteristics, where we live can also alter the degree to which earning a good wage can protect our health. Earning a decent wage, for example, does not necessarily ensure access to good services. Our local communities can offset or enhance the other resources we have available to us as we pursue health and longevity.

One consequence of focusing on ageing specifically is that it seems to obfuscate the inequalities that are producing the ageing crisis. In other words, the ageing crisis - the prospect of many elderly people living longer lives - will be especially challenging if the inequalities in ageing become even greater, with many more people suffering poor health while living longer lives. Moreover, it seems to overlook that the foundation for the inequalities in ageing that we observe are actually laid at other points during the life-course. The work of life-course epidemiologists has repeatedly shown us that ageing trajectories are forged in childhood, adolescence and our working lives. Minimizing the fiscal risks associated with an ageing population requires a life-course approach.

In the next chapter we show that acknowledging that the politics of ageing is really the politics of inequality is crucial because the policy solutions that are sometimes proposed to address aspects of the ageing crisis are inherently linked with inequality. Indeed, in some cases they may very well exacerbate health inequalities, making the ageing crisis worse in the long run. 